\title{
Ultrasonographic Evaluation of Sural Nerve for Nerve Conduction Study
}

\author{
Ki-Hoon Kim, MD, Ji-Yoon Yoo, MD, Byung-Chun You, MD
}

\author{
Department of Physical and Rehabilitation Medicine, Veterans Health Service Medical Center, Seoul, Korea
}

\begin{abstract}
Objective To understand various morphologic types and locations of the sural nerve (SN) that are important for nerve conduction studies or nerve grafting procedures. The aim of this study was to describe the course and variations of the SN based on ultrasonographic findings for an adequate nerve conduction study.

Methods A total of 112 SNs in 56 volunteers with no history of trauma or surgery were examined by ultrasonography. The location and formation of the SNs in relation to the medial and lateral sural cutaneous nerve were investigated. We measured the horizontal distance between the SNs and the midline of the calf at the level of $14 \mathrm{~cm}$ from the lateral malleolus, and the distance between the SNs and the most prominent part of the lateral malleolus.

Results SN variants was classified into four types according to the medial and lateral sural cutaneous nerve; type 1 (73.2\%), type $2(17.9 \%)$, type $3(8.0 \%)$, and type $4(0.9 \%)$. The mean distance between the SN and the midline of the calf was $1.02 \pm 0.63 \mathrm{~cm}$, the SN and the most prominent part of the lateral malleolus was $2.14 \pm 0.15 \mathrm{~cm}$.

Conclusion Variations in the location and formation of the SN was examined by ultrasonography, and the results of this study would increase the accuracy of the SN conduction study.
\end{abstract}

Keywords Sural nerve, Ultrasonography, Nerve conduction study

\section{INTRODUCTION}

The sural nerve (SN) is one of the major sensory nerves in the lower limb that provide cutaneous sensation to the lateral and posterior aspects of the distal third of the

Received July 31, 2013; Accepted September 2, 2013

Corresponding author: Ji-Yoon Yoo

Department of Physical and Rehabilitation Medicine, Veterans Health Service Medical Center, 53 Jinhwangdo-ro 61-gil, Gangdong-gu, Seoul 134-791, Korea

Tel: +82-2-2225-1535, Fax: +82-2-2225-1579, E-mail: miryjy@hanmail.net

(c) This is an open-access article distributed under the terms of the Creative Commons Attribution Non-Commercial License (http://creativecommons. org/licenses/by-nc/3.0) which permits unrestricted noncommercial use, distribution, and reproduction in any medium, provided the original work is properly cited.

Copyright $\odot 2014$ by Korean Academy of Rehabilitation Medicine leg, the lateral calcaneal region, the lateral aspect of the foot and the small toe. The SN originates just distal to the popliteal fossa, descends between the heads of the gastrocnemius, and pierces the deep fascia proximally in the leg. The medial sural cutaneous nerve (MSCN) emerges from the tibial nerve usually joined by the lateral sural cutaneous nerve (LSCN, peroneal branch) in the middle third of the calf, forming the final SN [1].

The SN has its importance in a number of clinical situations, including nerve conduction studies, nerve biopsies, and nerve graft procedures in the SN. Nerve conduction studies of the SN are widely used for the diagnosis of various types of polyneuropathies, and focal nerve injuries, such as lumbosacral plexopathy, sciatic and tibial neuropathies. However, anatomical variations 
of the SN, for example, variability in the course of the SN and in the union site between the MSCN and LSCN, may affect the latency and amplitude of sural sensory nerve action potential (SNAP), leading to misinterpretation of electrodiagnostic findings. Also, due to the proximity to the small saphenous vein (SSV), the SN is at risk of injury during dissection of the sapheno-popliteal junction, phlebectomy of the SSV and its tributaries, thermal ablation of SSV, or stripping of the SSV $[2,3]$.

The development of high-frequency broadband ultrasonography transducers has improved the ability of ultrasonography to image soft tissues. Ultrasonography can identify almost all the main nerves of the limbs. It has been reported on morphologic determination of SN types by ultrasonography or using cadavers [4-6]. However, ultrasonographic evaluations of the course and anatomical variations of the $\mathrm{SN}$ for nerve conduction studies have not been documented. The aim of this study was to identify the course and the anatomical variation of the SN based on ultrasonographic findings for an adequate nerve conduction study.

\section{MATERIALS AND METHODS}

In 2012 and 2013, a total of 112 SNs in 56 healthy subjects with no history of trauma or surgery underwent ultrasonographic examinations. Subjects with diabetes mellitus, nephrosis, peripheral neuropathy, and history of injury or surgery of the lower extremities were excluded from the study. This study protocol was approved by International Review Board of our hospital, and all the participants gave informed written consent for participation in this research.

Ultrasonographic scanning of the SN was performed by a single sonographer, who was experienced in musculoskeletal ultrasonography, using an ultrasonography machine Accuvix XQ (Medison, Seoul, Korea) with a linear 6-12 MHz high resolution transducer. Each subject was placed in the prone position with the knee fully extended and the ankle in the neutral position. The length of the leg from the fibular head to the lateral malleolus was measured. The ultrasonography transducer was first placed at the lower section of the calf. With the transverse image of the SSV, the SN usually appeared as a round or ovoid structure of 1-2 $\mathrm{mm}$ in diameter, moderately echogenic that contain black spots corresponding to nerve fibers surrounded by an echogenic sheath (epineurium) lying in close proximity to the SSV. Distally, the SN was detected between the Achilles tendon and the lateral malleolus. The transverse distance between the SN and the most prominent part of the lateral malleolus was measured as a recording point for the nerve conduction study. The transducer was then slowly moved proximally to obtain the union of the MSCN and LSCN, and then, the distance between the union and the lateral malleolus was measured. Next, the transducer was moved proximally along the MSCN and LSCN. If the union of the MSCN and LSCN was not detected, the imaging was moved toward the popliteal fossa to determine whether the SN was extended from the tibial nerve or the common peroneal nerve. The observed morphological formation of the SN from the MSCN or LSCN was used for the classification of the SN type.

Finally, we measured the distance between the SN and the mediolateral midpoint of the calf at the level of $14 \mathrm{~cm}$ from the lateral malleolus to allocate the stimulation site for the nerve conduction study. The distance would be a negative value if the SN lies medial to the midpoint of the calf. If the union of the SN was located below the level of $14 \mathrm{~cm}$ below the level from the lateral malleolus, the distances from the midpoint of the calf to the MSCN and to the LSCN were measured, respectively.

For statistical analysis, SPSS ver. 18.0 for Windows (SPSS Inc., Chicago, IL, USA) was used. The paired t-test was used to compare the location of the MSCN with that of the LSCN in type 1 at the level of $14 \mathrm{~cm}$ from the lateral malleolus. The independent sample t-test was used to analyze the difference between the course of the MSCN in type 2 and that of the LSCN in type 3. A p-value less than 0.05 was considered statistically significant.

\section{RESULTS}

The SN was identified using ultrasonography in all 56 subjects (112 limbs). We included 34 men and 22 women with a mean age of $37.5 \pm 9.3$ years (range, $22-$ 63 years). Their mean height was $168.0 \pm 6.7 \mathrm{~cm}$, and the mean weight was $60.6 \pm 8.15 \mathrm{~kg}$. The mean calf length was $37.16 \pm 2.34 \mathrm{~cm}$.

According to its formation and the course, the SN was assorted into four types. Type 1 consisted of formation of the SN with the union of the MSCN and LSCN (Fig. 1A). 

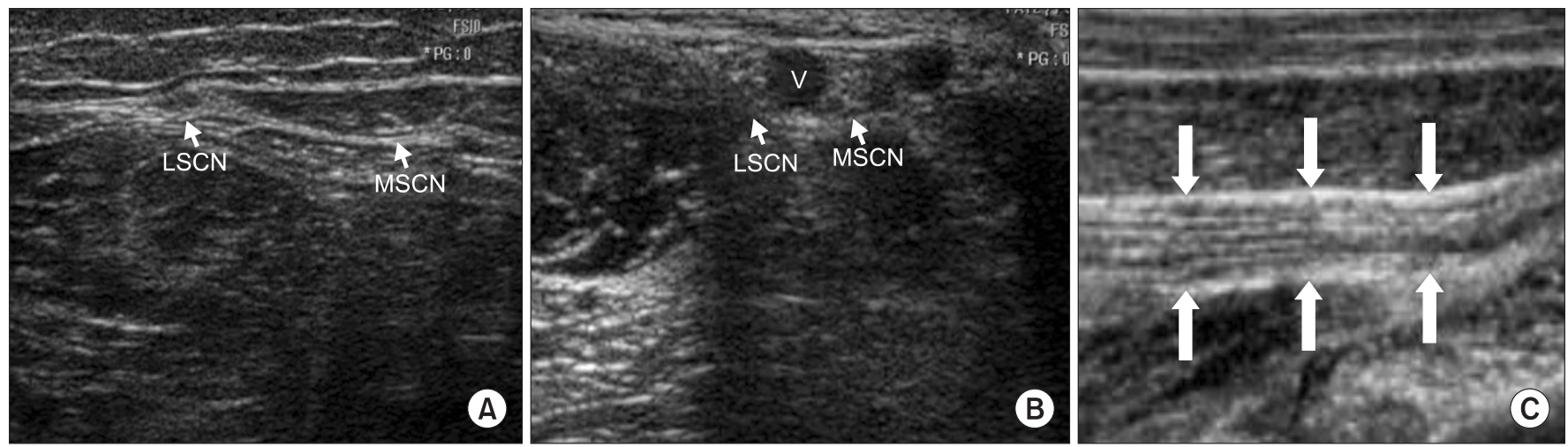

Fig. 1. Ultrasonography of a transverse view of sural nerve formation from medial sural cutaneous nerve (MSCN, right arrow) and lateral sural cutaneous nerve (LSCN, left arrow) at mid-calf level in type 1 (A), at ankle in type 4 (B). Longitudinal view of sural nerve (C). V, small saphenous vein.
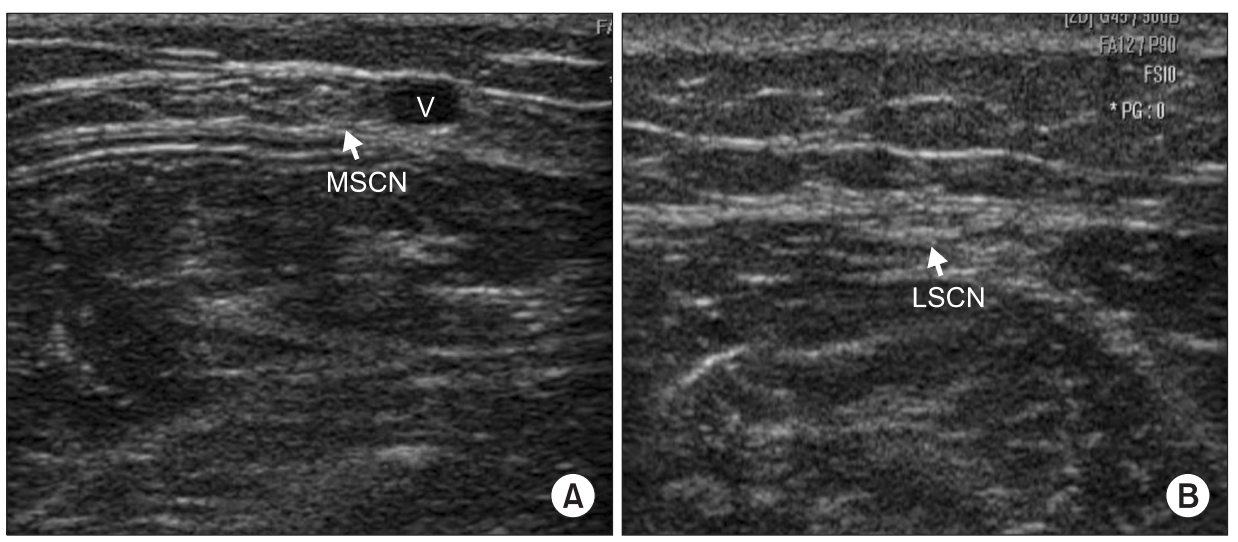

Fig. 2. Ultrasonography of a transverse view of sural nerve formation at mid-calf level in type 2 (A), at mid-calf level in type 3 (B). MSCN, medial sural cutaneous nerve; LSCN, lateral sural cutaneous nerve; $\mathrm{V}$, small saphenous vein.

The SN extending from the MSCN alone was relevant to type 2 (Fig. 2A), whereas the only LSCN was classified as type 3 (Fig. 2B). Type 4 consisted of MSCN and LSCN which descended down to the ankle separately (Fig. 1B). Eighty-two out of 112 legs were classified as type 1 (73.2\%), and the union sites of the SNs were located at $8.4 \pm 2.17 \mathrm{~cm}(36.95 \pm 11.66 \%$ of the length of lower leg) from the tip of lateral malleolus. Among type 1, $67.1 \%$ of the union sites were above the level of $14 \mathrm{~cm}$ from the lateral malleolus. Twenty legs were classified as type 2 (17.9\%). Nine legs were classified as type 3 (8.0\%). Only one leg was showed no communication and classified as type $4(0.9 \%)$.

The mean horizontal distance between the SN and the most prominent part of the lateral malleolus was $2.14 \pm 0.15 \mathrm{~cm}$, and the distances between the SN and the midline of the calf at the level of $14 \mathrm{~cm}$ from the lateral malleolus was $1.02 \pm 0.63 \mathrm{~cm}$. The distances according to the SN types and subtypes are shown in Table 1. At the level of $14 \mathrm{~cm}$ from the lateral malleolus, the LSCN of type 1 was located more laterally than the MSCN of the same type $(1.70 \pm 0.38$ vs. $0.71 \pm 0.35 \mathrm{~cm} ; \mathrm{p}<0.01)$, and the LSCN of type 3 had a more lateral position than the MSCN of type $2(1.69 \pm 0.67$ vs. $0.67 \pm 0.59 \mathrm{~cm} ; \mathrm{p}<0.01)$.

Furthermore, bilateral symmetry in the pattern of SN formation was found in 30 out of 116 legs (26.8\%), and similar to other previous studies, asymmetry is the rule rather than the exception [5]. However, there was no statistically significant difference between the left side and the right side.

\section{DISCUSSION}

The present study was conducted to identify the course and anatomical variations of the SN based on ultrasonographic findings for an adequate SN conduction study. Ultrasonographic evaluation showed type 1 classic formation of the SN (union of the MSCN and LSCN) in 
Table 1. Anatomical variations and the course of the sural nerve for the nerve conduction study based on ultrasonography

\begin{tabular}{|clll}
\hline Type & No. (\%) & Ds $(\mathbf{c m})$ & $\mathbf{D}_{\mathbf{R}}(\mathbf{c m})$ \\
\hline Type 1 & $82(73.2)$ & & \\
\hline $14 \mathrm{~cm}$ & 55 & $0.85 \pm 0.53$ & $2.11 \pm 0.09$ \\
\hline$<14 \mathrm{~cm}$ & 27 & & \\
\hline MSCN & & $0.71 \pm 0.35^{\text {a) }}$ & $2.21 \pm 0.23$ \\
\hline LSCN & & $1.70 \pm 0.38^{\text {a) }}$ & \\
\hline Type 2 & $20(17.9)$ & $0.67 \pm 0.59^{\text {b) }}$ & $2.11 \pm 0.09$ \\
\hline Type 3 & $9(8.0)$ & $1.69 \pm 0.67^{\text {b) }}$ & $2.12 \pm 0.13$ \\
\hline Type 4 & $1(0.9)$ & & \\
\hline MSCN & & $0.8 \pm 0.0$ & $2.69 \pm 0.0$ \\
\hline LSCN & & $1.2 \pm 0.0$ & $2.24 \pm 0.0$ \\
\hline
\end{tabular}

Values are presented as numbers (\%) or mean \pm standard deviation.

Ds, distances between the sural nerve and the midline of the calf at the level of $14 \mathrm{~cm}$ from the lateral malleolus; $\mathrm{D}_{\mathrm{R}}$, distances between the sural nerves and the most prominent part of the lateral malleolus; MSCN, medial sural cutaneous nerve; LSCN, lateral sural cutaneous nerve; type 1, the sural nerve with union of the MSCN and LSCN; type 2, extended from the MSCN alone; type 3 , continuation of LSCN; type 4, no communication between MSCN and LSCN.

${ }^{a)} \mathrm{p}<0.01$ by paired $\mathrm{t}$-test, ${ }^{\mathrm{b})} \mathrm{p}<0.01$ by independent $\mathrm{t}$-test.

$73.2 \%$ of the cases, type 2 (MSCN alone) in $17.9 \%$, type 3 (LSCN alone) in $8.0 \%$, and type 4 (no communication between MSCN and LSCN) in $0.9 \%$. The results also revealed that the $\mathrm{SN}$ had its course at a horizontal distance of $1.02 \pm 0.63 \mathrm{~cm}$ lateral to the midline of the calf at the level $14 \mathrm{~cm}$ from the lateral malleolus as the stimulation point for the nerve conduction study.

As earlier studies have reported, the SN has many variations in its formation, course, or in the union site between the MSCN and LSCN. In an anatomical study on the formation of the SNs in 76 cadavers, $67.1 \%$ of the SNs were formed by the union of the MSCN and LSCN, 32.2\% of the SNs by MSCN alone, and 1 SN was formed by the union of the MSCN and a different branch of the common peroneal nerve [4]. In another cadaver study with 26 legs, the SN was formed by the anastomosis of the MSCN and LSCN in 20 cases (76.9\%), the SN by the continuation of the MSCN alone in 4 cases (15.4\%), and no communication between the MSCN and LSCN in 2 cases (7.7\%) [5]. Although type 1 formation of the SN with the union of the MSCN and LSCN was the most frequent morphologic type of the $\mathrm{SN}$, there were some differences, such as the absence of the MSCN and the percentage of morphologic types between the present ultrasonographic study and earlier cadaver studies. These differences could be due to racial differences or the number of subjects studied. Also, differences between cadavers and living subjects could have altered the results. Cadaver studies could result in measurement errors because of the transposition of anatomic structures especially subcutaneous nerves or vessels during dissection. In contrast, ultrasonography is able to search for anatomic constructions without transposition.

The development of high-frequency broadband ultrasonography transducers has improved the ability of ultrasonography to image soft tissues including peripheral nerves. Ultrasonography can identify almost all the main nerves of the limbs, and has been successfully applied in the SN. The cross-sectional area of the SN in the distal calf was reported based on the intimate relationship between the lesser saphenous vein and the SN [7]. A study demonstrated that ultrasonographic guidance improved the quality of SN block as compared with the traditional landmark-based technique [8]. Ultrasonographic findings of the normal SN and its point of contact with the lesser saphenous vein was demonstrated [1]. The usefulness of ultrasonography to improve the positioning of the recording needle for $\mathrm{SN}$ conduction studies was also reported [9]. Ultrasonographic identification of the SN may also be useful before surgical Achilles tendon repair procedures, following which a $13 \%$ incidence of injury to the SN was reported [10].

The results of the current study are similar to those of a previous ultrasonographic study on anatomic variants of the SN which showed that type 1 formation as the union of MSCN and LSCN was found in $81 \%$ of the cases, type 2 by MSCN alone in $18 \%$, and type 3 by LSCN alone in $1 \%$ of the SNs [6]. The present study demonstrated more cases of type 3 by LSCN alone and also documented one leg of additional morphologic type 4 of the SN with no communication between the MSCN and LSCN. Furthermore, the current ultrasonographic study evaluated the pathway of the SN as stimulation and recording sites for nerve conduction studies.

An electrophysiology study reported that the largest SNAP was evoked by stimulation at a point $1 \mathrm{~cm}$ lateral 
to the midline of the calf, $14 \mathrm{~cm}$ proximal to the midpoint between the lateral malleolus and the Achilles tendon [5]. The location of the stimulation point resembled the SN pathway investigated at the level of $14 \mathrm{~cm}$ from the lateral malleolus by ultrasonography in our study.

The site of union of the MSCN and LSCN is known to be highly variable. An ultrasonographic study reported that the SN had a length of $10-20 \mathrm{~cm}$ in $74.5 \%$ of the type 1 SNs [6]. Another study using cadavers reported that the union occurred in the distal third of the leg (67.4\%) [4]. In the present study, the union of the MSCN and LSCN was observed most frequently in the middle third of the leg $(67 \%)$. The variability in the union site between the MSCN and LSCN can affect the parameters used in the SN conduction study. If the MSCN and LSCN are separated or the union of the two nerves is distal to the standard stimulation point, the MSCN or LSCN could be stimulated alone, resulting in low sural SNAP or in the case of simultaneous stimulation of the two nerves, SNAPs of double peaks can be generated. Pyun and Kwon [5] showed separate SNAPs of the MSCN and LSCN in 4 legs among 30 healthy subjects, and recorded a double peak in each of 4 legs.

Interestingly, in contrast to the present study and the recent ultrasonographic study, no previous cadaver studies showed morphologic types of the $\mathrm{SN}$ formed from the LSCN alone. Although the reason of this is not clear, there is a possibility that the MSCN with a small diameter could have been missed on the ultrasonography screen, or the LSCN, despite its existence, might not be traced proximally to the common peroneal nerve in the cadaver studies.

The present study has several limitations. First, the small sample size might have influenced the results especially in the detection of rare morphologic types of the SN. Second, verification of the SN type from the nerve conduction study was not performed. Third, it was difficult to measure the size of the SN due to its small diameter.

Despite these limitations, this study demonstrated the optimal stimulation point in the SN conduction study and identified the SN pathway and its various types for the classification of the SN by ultrasonography.

In conclusion, anatomical variations in the location and formation of the SN were examined by ultrasonography. The SN is formed from various combination types of the MSCN and LSCN. The SN pathway as the stimulation point in the nerve conduction study was investigated by ultrasonography and was located about $1 \mathrm{~cm}$ lateral to the midline of the calf at the level of $14 \mathrm{~cm}$ from the lateral malleolus. These findings would increase the accuracy of the SN conduction study. If the sural SNAPs have appreciable side-to-side distinction in the amplitude or revealed low amplitude in spite of normal neurologic evaluation, the possibility of anatomical variation should be considered.

\section{CONFLICT OF INTEREST}

No potential conflict of interest relevant to this article was reported.

\section{REFERENCES}

1. Ricci S, Moro L, Antonelli Incalzi R. Ultrasound imaging of the sural nerve: ultrasound anatomy and rationale for investigation. Eur J Vasc Endovasc Surg 2010;39:636-41.

2. Sam RC, Silverman SH, Bradbury AW. Nerve injuries and varicose vein surgery. Eur J Vasc Endovasc Surg 2004;27:113-20.

3. Simonetti S, Bianchi S, Martinoli C. Neurophysiological and ultrasound findings in sural nerve lesions following stripping of the small saphenous vein. Muscle Nerve 1999;22:1724-6.

4. Mahakkanukrauh $\mathrm{P}$, Chomsung R. Anatomical variations of the sural nerve. Clin Anat 2002;15:263-6.

5. Pyun SB, Kwon HK. The effect of anatomical variation of the sural nerve on nerve conduction studies. Am J Phys Med Rehabil 2008;87:438-42.

6. Zhu J, Li D, Shao J, Hu B. An ultrasound study of anatomic variants of the sural nerve. Muscle Nerve 2011;43:560-2.

7. Cartwright MS, Passmore LV, Yoon JS, Brown ME, Caress JB, Walker FO. Cross-sectional area reference values for nerve ultrasonography. Muscle Nerve 2008;37:566-71.

8. Redborg KE, Sites BD, Chinn CD, Gallagher JD, Ball PA, Antonakakis JG, et al. Ultrasound improves the success rate of a sural nerve block at the ankle. Reg Anesth Pain Med 2009;34:24-8.

9. Kamm CP, Scheidegger O, Rosler KM. Ultrasound- 
guided needle positioning in sensory nerve conduction study of the sural nerve. Clin Neurophysiol 2009;120:1342-5.
10. Webb J, Moorjani N, Radford M. Anatomy of the sural nerve and its relation to the Achilles tendon. Foot Ankle Int 2000;21:475-7. 